\title{
A structured approach to microcomputer system design
}

\author{
KEN McKENZIE \\ Intel Corporation, Santa Clara, California 95053
}

\begin{abstract}
A simple "building block" philosophy for construction of high-performance microcomputer-based systems utilizing I/O functional definition and a structured implementation of MOS/LSI semiconductor devices.
\end{abstract}

The wide acceptance of the microcomputer as a practical tool for on-line computation and dedicated control applications has drastically altered the manner in which today's design engineer approaches a new project. The traditional circuit by circuit analysis is no longer necessary or desirable. A total systems approach of closely studying each $\mathrm{I} / \mathrm{O}$ device and its interface in conjunction with the desired performance level of the overall system then, by selecting the proper MOS/LSI devices and writing the support software, produces an efficient, modular design.

The purpose of this paper is to show how a building block approach to both hardware and software will effect system design, introduce the latest MOS/LSI devices, and familiarize the uninitiated engineer with basic microcomputer terminology and jargon.

The structured philosophy presented here is based on the 8080 Microcomputer System (MCS-80), Intel Corporation, Santa Clara, California. ${ }^{1}$

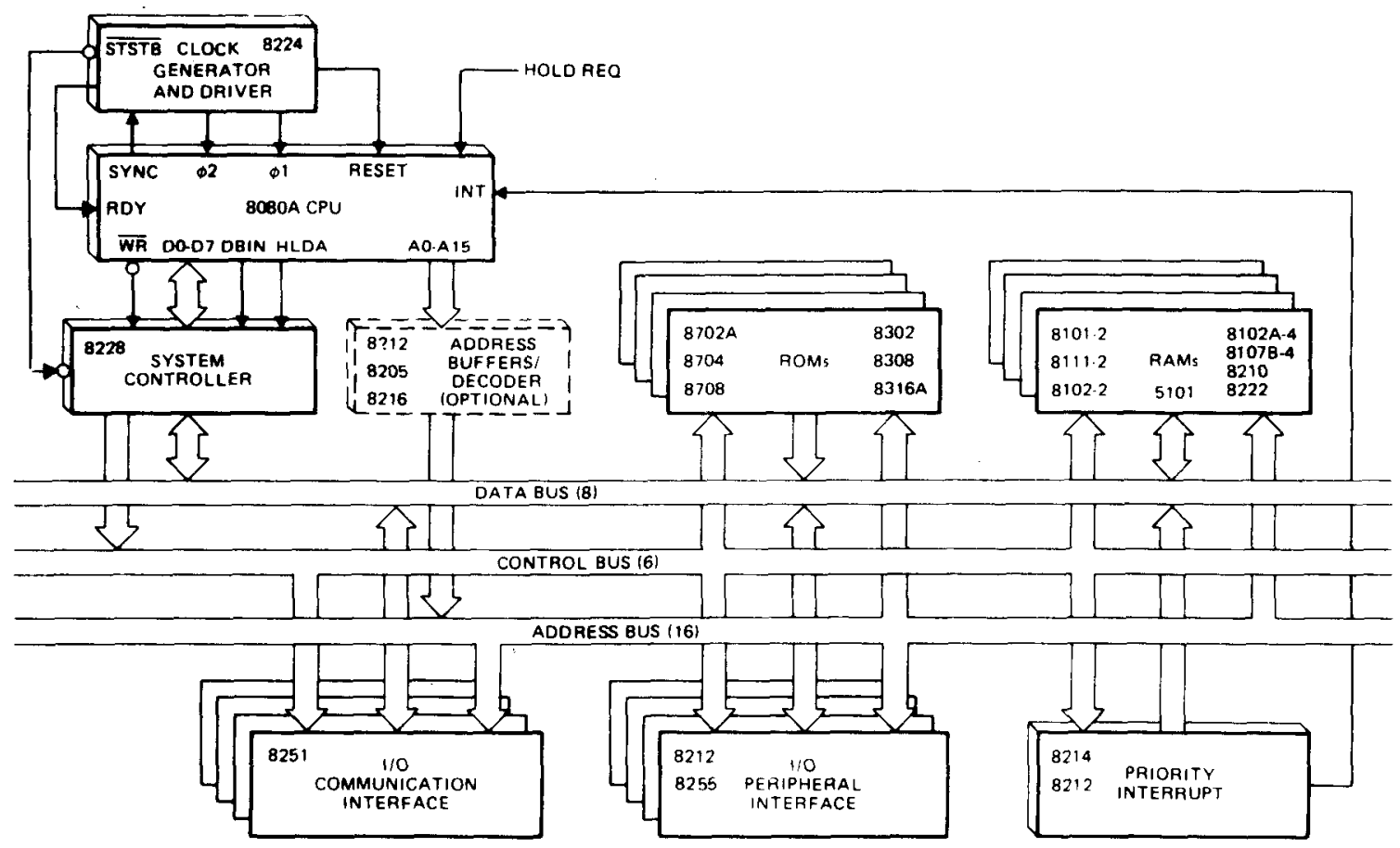

Figure 1. Microcomputer system (courtesy of Intel Corporation, Santa Clara, California).
The heart of any structured philosophy is the discipline upon which the system is based. In a microcomputer system, this discipline is referred to as the system bus. Basically, it is a group of interconnections that carry the total information transfer from one device in the system to another. All system devices, central processing unit, memory. and $\mathrm{I} / \mathrm{O}$, intercommunicate over the system bus.

In the 8080 microcomputer system (see Figure 1), the system bus is broken down into three major groups: Data bus is the bus upon which all data flows in the system. It is bi-directional in nature, allowing both read and write operations to utilize the same bus. Address bus: When the CPU wishes to read or write to a specific memory or $\mathrm{I} / \mathrm{O}$ device, it issues a linique binary value (address) on the address bus. Control bus: The collection of timing signals that gates data on and off of 


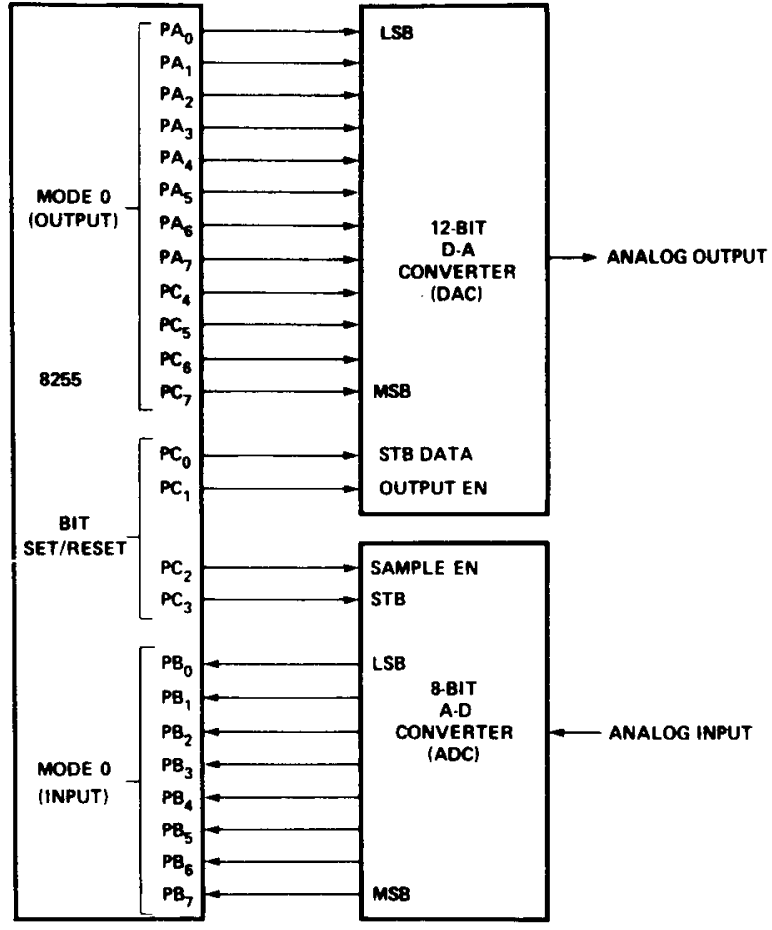

Figure 2. Digital to analog; analog to digital (courtesy of Intel Corporation, Santa Clara, California.

the data bus is called the control bus. These signals are precisely generated so that when the transfer occurs, the data is stable and no conflicts occur with other devices on the system bus.

The transfer of information over the system bus must abide by an established procedure, which is called protocol. Bus protocol dictates a sequence of events that any device on the system bus must follow for efficient data transfer. The timing disciplines of the bus protocol establishes "windows" in which the peripheral device must respond with valid data or be prepared to accept valid data. There are auxiliary functions of the protocol that can insert compensation factors into these timing windows, such as "wait states." Consequently, the protocol discipline is designed to contain a limited degree of flexibility.

The establishment of a standard system bus architecture greatly simplifies the design of peripheral, semiconductor components for microcomputer systems. The interface to the microprocessor-generated data, address, and control buses remains identical, and only the individual component functions are subject to additional circuit engineering. Besides timing (ac characteristics), the system bus also defines dc characteristics such as leakage, current drive, voltage levels, and capacitance. An often underrated feature, inherent in a properly defined standard system bus, is overall system noise immunity. The real measure of cost effectiveness of any system is the amount of time that such a system can be operated and provide accurate information. By allowing for adequate system tolerance to noise in the design of the system bus, a high-performance, reliable system can
Table 1

MCS-80 Component Family*

\begin{tabular}{cl}
\hline Part No. & \multicolumn{1}{c}{ Function } \\
\hline $8080 \mathrm{~A}$ & CPU \\
8224 & Clock Generator \\
8228 & System Controller \\
8212 & Byte I/O \\
8251 & Communication Interface \\
8255 & Programmable I/O \\
8253 & Programmable Interval Timer \\
8257 & Programmable DMA Controller \\
8259 & Programmable Interrupt Controller \\
\hline *MCS-80 uses standard semiconductor memory components.
\end{tabular}

be inplemented using a family of bus-compatibie, microcomputer, peripheral components.

\section{I/O DEVICE INTERFACE}

As in any computer-based system, the microprocessor must be able to communicate with devices or structures that exist outside of its normal memory array. Devices such as keyboards, switches, displays, floppy disks, printers, A/D-D/A converters, and other control structures are used to input information into the microprocessor and store or display results of the computational activity (see Figures 2 and 3 ).

The basic operation of the $\mathrm{I} / \mathrm{O}$ structure of a microcomputer system can best be viewed as an array of single byte memory locations that can be read from or written into. Generally, there are special instructions within the basic repertoire to handle such transfers (IN, OUT). The CPU issues a unique binary code, corresponding to the particular I/O port with which it wishes to communicate. The data bus is made available to the selected device, and the transfer occurs in accordance with the discipline of the bus protocol. An array of switches, for example, can be monitored by the microprocessor by simply assigning each switch as a single bit of an input "port." The CPU can then read the value of the switch array under control of the system software. In turn, the microprocessor can output the results of its computation to an array of lights so that it can be read and interpreted by the system user. These simple examples are, of course, descriptive of only the most primitive $\mathrm{I} / \mathrm{O}$ functions of a microcomputer system. More common I/O applications would be driving motors, scanning keyboards, and monitoring analog sensors. Quite often the microcomputer is required to communicate with other computer systems that are separated by a great distance. By converting parallel system data (bytes) to a serial stream of data, the microcomputer system can use voicegrade telephone lines for this communication. Special semiconductor devices are specifically designed to handle both transmission and reception of serial formatted data; such a device is the Intel 8251 (Figure 4).

The recent advances in MOS/LSI technology, mainly 


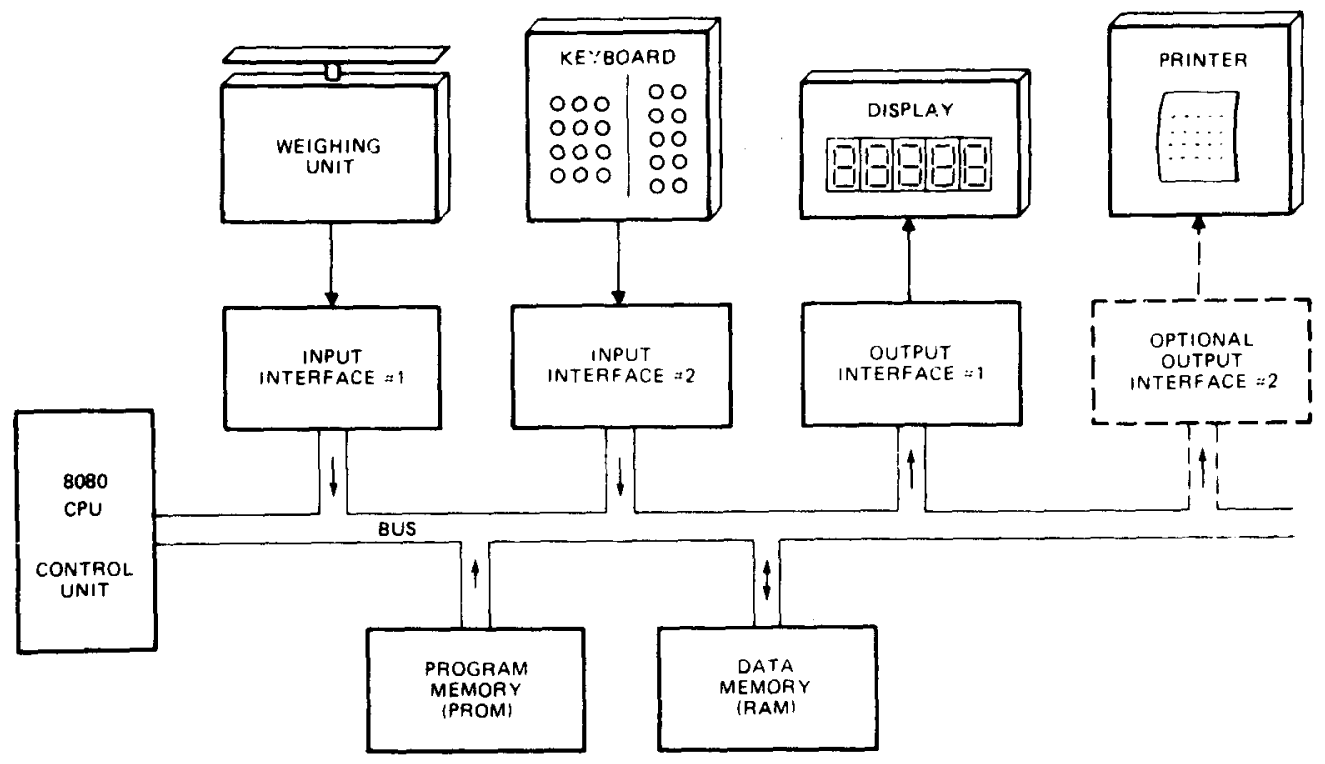

Figure 3. Data bus (courtesy of Intel Corporation, Santa Clara, California).

$\mathrm{N}$-channel MOS, has made dedicated peripheral and $\mathrm{I} / \mathrm{O}$ device controllers an economic reality. The complex nature of modern peripheral equipment interface and the resultant software overhead has made the use of such equipment unattractive to the small system user. By designing a certain amount of "intelligence" in to the semiconductor components that interface such equipment to the microcomputer system, the amount of software overhead can be significantly reduced and the number of chips decreased, often to a single LSI component. Naturally, all such components would have to be designed to the system bus specification for simple interface, but beyond that, each MOS/LSI component becomes a "building block" whose only difference to any other "building block" in the I/O structure is its functional definition. The system software also must undergo a change in philosophy. Each MOS/LSI peripheral component can be programmed to support a variety of configurations within its major functional definition. The software must initialize such devices and supervise whatever maintenance is required for their operation. It be-

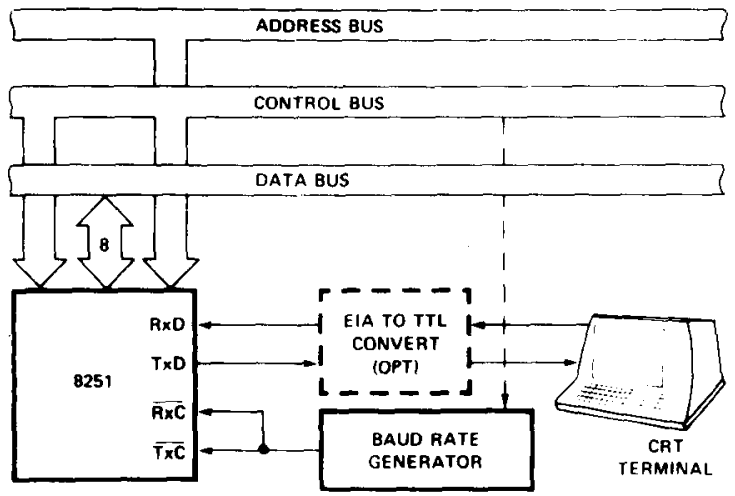

Figure 4. Asynchronous serial interface to CRT terminal. DC-9600 baud (courtesy of Intel Corporation, Santa Clara. California). comes logical to organize the systems and application software into a straightforward, modular fashion, almost "building block" software. The modular approach to software generation also yields inherent diagnostic capabilities and greater responsibility.

\section{INTERRUPTS}

The real efficiency of the microcomputer is directly proportional to the amount of tasks it can assume with little or no effect on the total system throughput. The most common method for $\mathrm{I} / \mathrm{O}$ management is the polled approach (Figure 5). Each $1 / 0$ device in the system is periodically tested by the software to ascertain if it needs servicing, commonly referred to as "polling" an $\mathrm{I} / \mathrm{O}$ device. It is easy to see that a significant portion of

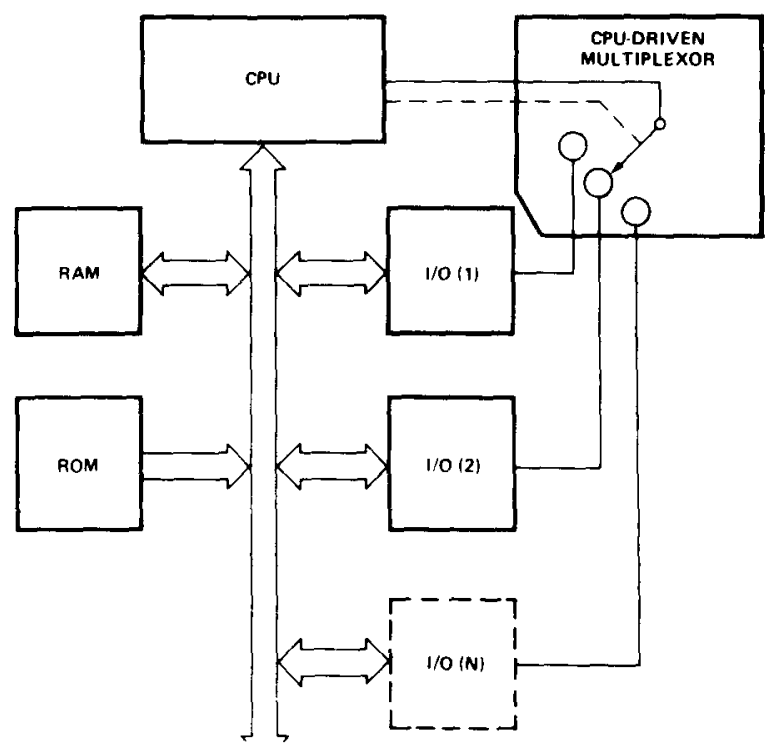

Figure 5. Polled method (courtesy of Intel Corporation, Santa Clara, California). 


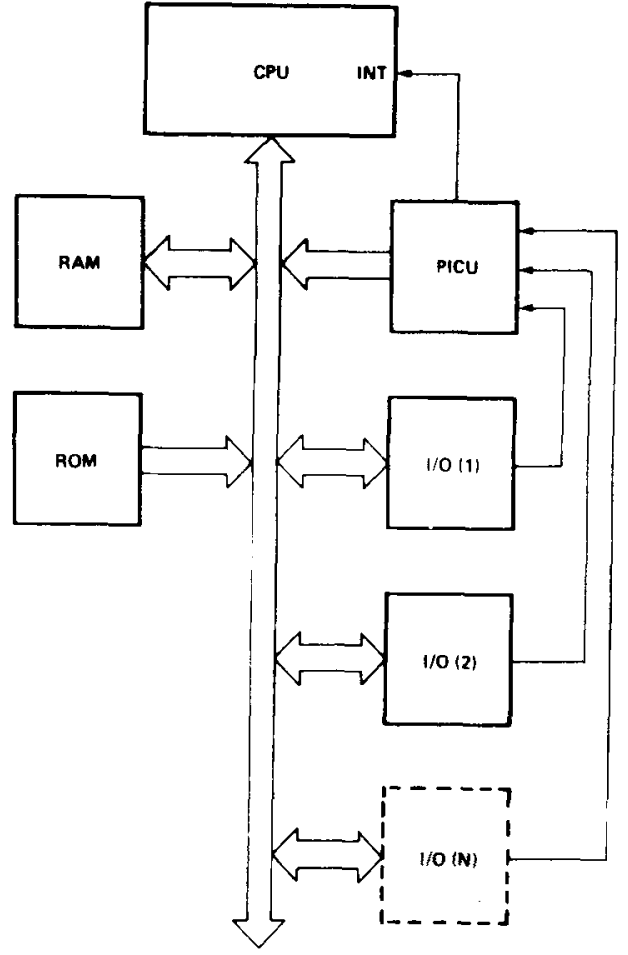

Figure 6. Interrupt method (courtesy of Intel Corporation, Santa Clara, California).

the program would be devoted to managing this overhead and result in a decrease in system throughput. A more desirable method would be one that would allow the microprocessor to be executing its main program and only stop to service peripheral devices when it is told to do so by the device itself. In effect, the method would provide an external, asynchronous input that informs the processor to complete whatever instruction is currently being executed and fetch a new routine that will service the requesting device. Once this servicing is complete, however, the processor would resume exactly where it left off. This method is called interrupt (see Figure 6). It is easy to see that system throughput would drastically increase, and hence more tasks could be assumed by the microcomputer to further enhance its efficiency and cost effectiveness.

In an interrupt-driven system, it is common that a number of peripheral devices would be requesting the attention of the processor, some at the exact same time. To efficiently manage such requests, the system designer must assign each requesting device a degree of importance, or level of priority, as it is more commonly referred to.

An auxiliary component, called an interrupt control unit, does the actual prioritizing and management of the incoming requests. The system designer simply connects each device's request lines to the PICU in the order of importance that he determines. The PICU monitors all incoming requests, ascertains which has the highest priority, and issues a composite request to the CPU along with information that can help the CPU identify the requesting device. Such a priority interrupt control unit is the INTEL 8214 (see priority interrupt, Figure 1). The latest semiconductor components that are dedicated to managing interrupts are complex MOS/LSI devices that actually do a small amount of preprocessing of the requests. For example, the 8259 of Intel (Figure 7) is an eight-level controller that is programmed by the system software to select from an array of service algorithms for more efficient interrupt management. With complete software control of the algorithms by which the incoming requests are handled, the interrupt can be changed dynamically as the total system environment changes.

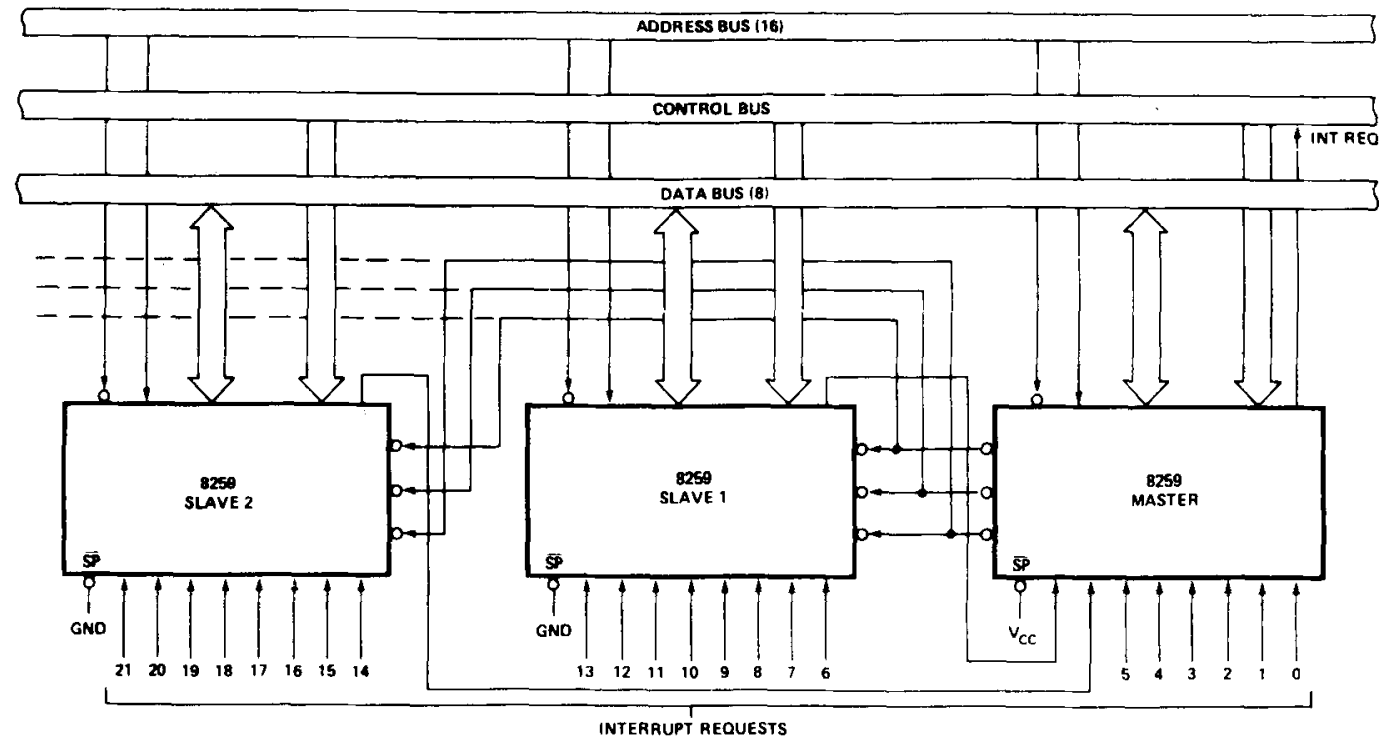

Figure 7. The 8259 eight-level controller (courtesy of Intel Corporation, Santa Clara, California). 


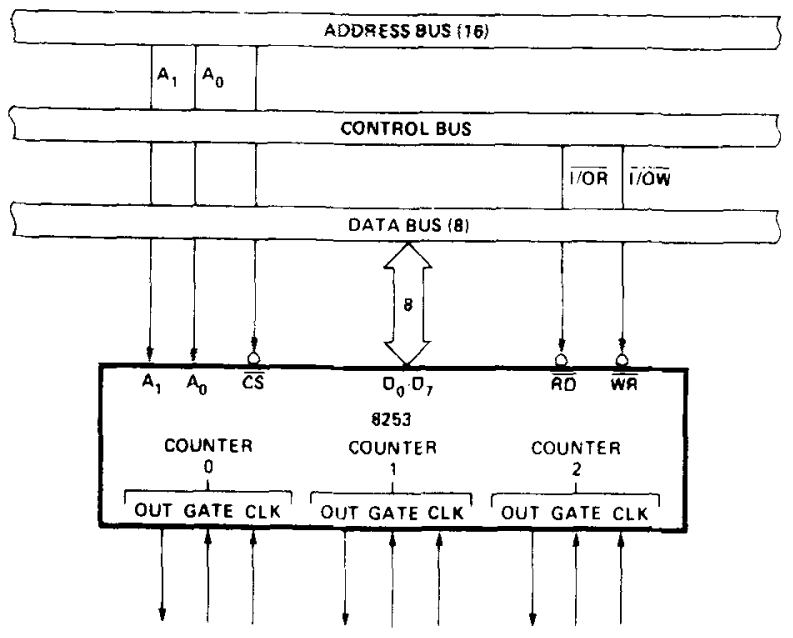

Figure 8. The 8253 system interface (courtesy of lntel Corporation, Santa Clara, California).

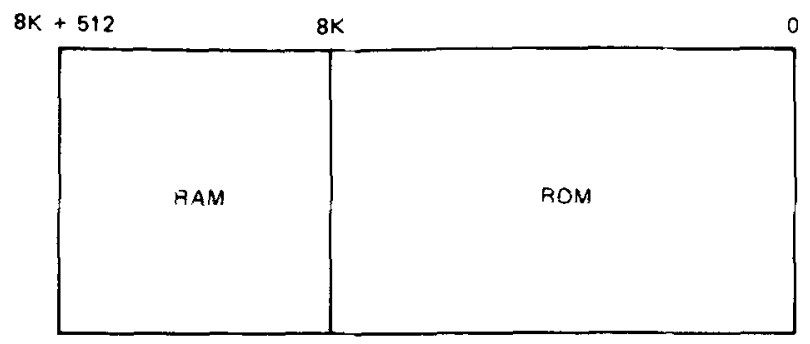

MEMORY MAP

Figure 9. Memory map (courtesy of Intel Corporation, Santa Clara, California).

\section{LSI INTERVAL TIMER}

One of the most common problems in microcomputer system design is interfacing to slow, delaydependent peripherals devices such as: stepper motors. hammer relays, and other such mechanical devices. The system's software allows for such delays by programming timing loops. This type of programming requires significant overhead, and the maintenance of multiple loops gets extremely complicated, especially in an interrupt-driven environment. The Intel 8253 is a single chip solution to most system timing problems (Figure 8), In essence, $\mathrm{jt}$ is a collection of three 16-bit counters that are totally independent in nature but driven commonly as $\mathrm{I} / \mathrm{O}$ peripheral ports. Instead of setting up timing loops in software, the programmer configures the 8253 to match his requirements. The programmer initializes the 8253 with quantity of the count and desired mode. then, upon command, the 8253 counts out the delay and interrupts the microprocessor when it has finished its tasks. It is easy to see that the software overhead is minimai and that muitiple delays can easily be main. tained by assignment of interrupt levels to different counters. Other functions that can be accomplished with the 8253 interval timer are nondelay in nature: baud rate generator, event counter, binary rate multiplier, and real-time clock.

The 8253 represents a significant improvement for solving one of the most common problems in system design and software generation.

\section{SYSTEM MEMORY CONSIDERATIONS}

Generally, there are two types of memory storage: read only (ROM) and read/write (RAM). The ROMs provide for nonvolatile storage. so that the main program will not be lost when the system power is shut off RAMs provide temporary storage for intermediate data and semi-permanent storage for dynamically alterable software. The interface of either type of storage to the standard system bus is very similar to that of the $/ / 0$ devices. It is best to view mernory as groups of building blocks all structured within the disciplines of the standard system architecture. A simple memory "map" can be developed that shows the area of address space that each type of memory occupies to further enhance the simple, block approach of system design. (Refer to Figures 9 and 10 .)

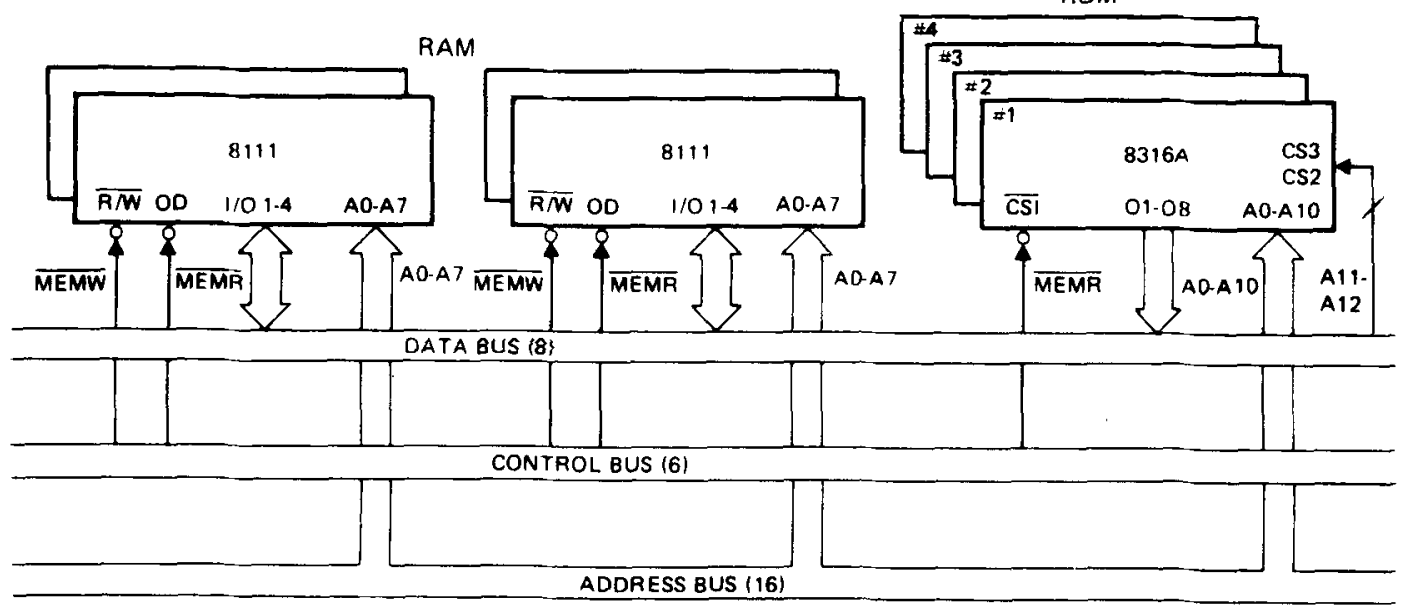

Figure 10. Typical memory interface (courtesy of Intel Corporation. Santa Clara, California). 


\section{CONCLUSION}

The microcomputer has gained wide acceptance as a practical tool for solving complex problems. To expand the spectrum of applications, a total systems philosophy that allows for simple implementation, fast construction, and inherent reliability is a must. A logical structured approach to system design that relies on complete functional definition and component implementation of the problem yields a cost-effective, reliable solution with absolutely no sacrifice on performance.
Advances in MOS/LSI technology will determine what the future of the microcomputer system will be. As more complex peripheral components become available, the microprocessor becomes simply a manager of the $I / O$ structure, and the real challenge is the imagination of the system designer and programmer to create efficient solutions to tomorrow's problems.

\section{NOTE}

1.8080 User's Manual, September 1975, Intel Corporation, Santa Clara, California 95053. 